\title{
O Excesso de Confiança dos Produtores de Milho no Brasil e o Uso de Contratos Futuros
}

\author{
José César Cruz Júnior ${ }^{1}$ \\ Scott H. Irwin ${ }^{2}$ \\ Pedro Valentim Marques ${ }^{3}$ \\ João Gomes Martines Filho ${ }^{4}$ \\ Mirian Rumenos Piedade Bacchi ${ }^{5}$
}

Resumo: $\mathrm{O}$ objetivo deste artigo foi identificar sinais de excesso de confiança nos preços entre produtores de milho do Sul e do Centro-Oeste do Brasil. Entre outubro e novembro de 2008, 90 produtores foram selecionados para responderem questóes relacionadas a seus conhecimentos do mercado futuro e a suas expectativas de preços. Uma grande parte dos entrevistados respondeu que não negociava contratos futuros por não possuir informação suficiente para isso. Os resultados revelaram que os produtores foram descalibrados quando estimaram os preços esperados na forma direta e indireta. Além disso, para a maior parte dos respondentes, a variância subjetiva obtida por meio dos questionários foi estatisticamente inferior à variância do mercado. Isto mostra que os produtores possuem uma percepção de risco inferior ao risco de mercado. Por fim, o artigo conclui que o efeito de excesso de confiança pode, parcialmente, explicar o baixo uso do mercado futuro de milho por parte dos produtores brasileiros, para garantir a proteção de preço do produto.

Palavras-chave: excesso de confiança, probabilidade subjetiva, mercados futuros.

1 Professor do Departamento de Economia da Universidade Federal de São Carlos, Campus Sorocaba. E-mail: cesarcruz@ufscar.br

2 Laurence J. Norton Chair of Agricultural Marketing, Department of Agricultural and Consumer Economics, University of Illinois at Urbana-Champaign. E-mail: sirwin@uiuc.edu

3 Professor do Departamento de Economia, Administração e Sociologia da ESALQ/ USP. E-mail: pvmarque@usp.br

4 Professor do Departamento de Economia, Administração e Sociologia da ESALQ/ USP. E-mail: martines@usp.br

5 Professora do Departamento de Economia, Administração e Sociologia da ESALQ/ USP. E-mail:mrpbacch@esalq.usp.br 


\begin{abstract}
This paper aimed to identify signs of overconfidence among corn producers in the Southern and Central-Western regions in Brazil. Between October and November 2008, 90 farmers were chosen to answer questions regarding their knowledge of futures markets and price expectations. Most part of agents surveyed answered that they do not trade futures contracts because they do not have enough information. Results showed that respondents were miscalibrated when estimating directly-stated and the indirectly-stated expected prices. In addition, for most respondents, subjective variance for corn proves to be significantly less than the market variance, implying that producers perceive a risk that is lower than that of the market. Finally, the paper concludes that the overconfidence effect can partially explain the low use of futures markets by Brazilian corn producers to hedge their production.
\end{abstract}

Key-words: overconfidence, subjective probability, futures markets.

Classificação JEL: Q13, P14.

\title{
1. Introdução
}

A atividade agropecuária se depara com diversos tipos de risco, tais como: de preço, clima, crédito, custos, operacional etc. Entre estes, o preço da commodity é uma variável importante na determinação do sucesso ou não da atividade. Desta forma, um gerenciamento eficiente do risco de preço é crucial para que a atividade de longo prazo seja sustentável, e muitos instrumentos existem no mercado para auxiliar nesta decisão (EGELKRAUT et al., 2006).

Aguiar (1999) mostrou que existem ao menos três estratégias que podem ser utilizadas por agricultores no intuito de gerenciar o risco. São elas: o mercado a termo, o de futuros e o de opções. Dependendo do objetivo do produtor, cada uma dessas alternativas traz mais vantagens do que a outra.

Se, por um lado, estas estratégias são conhecidas como importantes ferramentas de gerenciamento de preços nos mercados agrícolas, por outro lado, na prática, elas não são amplamente utilizadas pelos produtores brasileiros (BORELLA, 2004). Em uma pesquisa realizada pela Associação Nacional dos Confinadores - Assocon (2008), descobriu-se que apenas $4 \%$ dos 486 confinadores de boi gordo no estado de Goiás protegem sua produção contra variações de preços na Bolsa de Mercadorias, Valores \& Futuros - BM\&FBovespa ${ }^{6}$.

Pelo ponto de vista acadêmico, muitos estudos já apontaram os benefícios que os produtores podem obter quando utilizam essas ferramentas, principalmente os mercados futuros (MARQUES, MELLO e MARTINES

6 De acordo com o IBGE (2008), em 2007, o estado de Goiás era o quarto mais importante produtor de boi gordo no Brasil. 
FILHO, 2008; ALVES, DUARTE e LIMA, 2008 e SILVEIRA e FERREIRA FILHO, 2003; ARBEX e CARVALHO, 1999). Entretanto, somente alguns trabalhos preocuparam-se em explicar a pouca utilização dessas ferramentas pelos produtores. Andrade (2004), por exemplo, mostrou que os custos de transação nos mercados futuros brasileiros podem explicar a razão pela qual muitos produtores de milho não realizam negócios na BM\&FBovespa para protegerem sua produção contra oscilações desfavoráveis de preços. Este mesmo autor mostrou ainda que os custos de transação são, em média, entre $2 \%$ e 6,5\% do valor inicial do contrato. Ele concluiu que o risco de custos provoca uma redução nos benefícios da administração de preços oferecidos pelos mercados futuros que é similar ao risco de base. Assim sendo, o risco de custo pode reduzir o desejo de produtores em fazer o hedge. Cruz Júnior, Marques e Martines Filho (2008) demonstraram que, considerando-se que o risco pode ser encarado como os desvios abaixo de um determinado retorno-alvo (geralmente a parte negativa da distribuição de probabilidades dos retornos de um investimento - downside risk), a "verdadeira" razão de hedge pode ser menor que a razão de hedge tradicional, obtida pelo modelo padrão de média e variância. Tais autores concluíram que, pelo simples fato de encararem o risco de uma forma alternativa e mais realista, quanto mais aversão ao risco tiver o produtor de boi gordo, menor poderá ser a parcela da produção que o mesmo terá hedgeada no mercado futuro da BM\&FBovespa.

Desta forma, pode-se afirmar que alguns autores já se atentaram para o relativo baixo uso de ferramentas de proteção de preços por parte dos agricultores brasileiros. No entanto, até o presente momento não pôde ser encontrado, na literatura brasileira, trabalhos nos quais alguma atenção teórica ou empírica tenha sido dada para a relação entre os preços agrícolas cotados em mercados futuros e as expectativas subjetivas dos indivíduos.

Nenhuma atenção ainda foi dada à expectativa subjetiva dos produtores em relação aos preços futuros ou às influências psicológicas na análise decisória dos mesmos. Como discutido por Eales et al. (1990), este assunto é de grande importância para a validação de muitos estudos que assumem que o preço futuro é uma proxy para expectativa de preços, pois essa relação é fundamental para muitas decisões de hedge. Por exemplo, se um produtor for otimista em relação ao preço futuro/spot, ele não terá nenhuma razão para assumir uma posição short (vendida) no mercado futuro.

Esse otimismo pode ser percebido em pelo menos duas formas diferentes: i) espera-se que os preços futuros sejam mais elevados do que a média histórica, e/ou ii) espera-se que a variância dos preços futuros seja inferior à variância histórica. Esse otimismo é referido na literatura como "excesso de confiança" (overconfidence) e pode ser considerado um ponto fundamental na explicação do motivo pelo qual produtores fazem proteção de preços para apenas uma pequena parcela de sua produção ou simplesmente não fazem o hedge. 
A motivação desse trabalho é verificar se os produtores brasileiros de milho exibem algum sinal de excesso de confiança em relação a preços. Com a não rejeição dessa hipótese, pode-se lançar uma explicação alternativa às explicações até agora encontradas na literatura nacional para determinar as razões pelas quais produtores não fazem hedge no mercado futuro.

\section{Revisão de Literatura}

A relação entre o preço futuro e o preço spot esperado de uma commodity no futuro é um tema já há muito tempo discutido na literatura econômica. De acordo com Bodie, Kane e Marcus (2001), este assunto gera bastante controvérsia e as discussões a respeito deste tema podem ser agrupadas em três diferentes teorias que fazem parte da Moderna Teoria de Porfólios, sejam elas: a teoria das expectativas, normal backwardation e contango.

De acordo com a hipótese das expectativas, o lucro esperado em uma posição assumida no mercado futuro, seja ela comprada ou vendida, é zero. Tal hipótese baseia-se na idéia de que os indivíduos são neutros ao risco, e assemelha-se ao equilíbrio de mercado em um ambiente sem risco. Desta forma, esta teoria pode ser criticada por não considerar o prêmio de risco existente no preço futuro devido à incerteza do preço spot esperado da commodity.

As duas outras teorias, normal backwardation e contango, consideram a existência do prêmio pelo fato de que especuladores não desejariam negociar contratos futuros a menos que pudessem lucrar com a negociação. Ou seja, tais agentes requerem uma compensação pelos riscos que carregam ao assumir uma posição no mercado. Os hedgers, por outro lado, estariam preparados para aceitar resultados negativos na negociação por causa dos benefícios da redução do risco que a posição nos mercados futuros pode proporcionar (HULL, 2005). Se, por exemplo, os hedgers tendem a manter posições vendidas no mercado futuro, para que os especuladores assumam posições compradas, os hedgers devem oferecê-los uma expectativa de lucro (prêmio pelo risco). Assim, os especuladores entrarão no mercado, tomando o risco dos hedgers, se o preço futuro estiver abaixo do preço spot esperado da commodity (incerto).

A teoria do normal backwardation sugere que o preço futuro será inicialmente ofertado a um nível abaixo do preço spot esperado e subirá ao longo da vida do contrato até a data de vencimento, quando os dois preços convergirão. Uma vez que também existem hedgers que assumem posições compradas no mercado futuro, a situação contrária também pode acontecer. Desta forma, a situação em que o preço futuro excede o preço spot esperado é conhecida como contango (BODIE, KANE e MARCUS, 2001).

Embora estas teorias tenham contribuído significativamente para a evolução das discussões e desenvolvimento de modelos que descrevem o comportamento 
dos preços, elas não abordam explicitamente o comportamento dos tomadores de decisão no momento em que assumem as posições no mercado futuro. $\mathrm{O}$ comportamento de excesso de confiança (overconfidence) tem sido citado por muitos trabalhos na tentativa de explicar a tomada de decisão individual diante de incerteza, sem encontrar-se diretamente relacionado a nenhuma das teorias descritas anteriormente. Cunningham III et al. (2008), por exemplo, encontraram que o excesso de confiança entre os homens faz com que eles realizem negócios com mais frequência que as mulheres no mercado de trigo. Apesar de não terem encontrado diferenças significativas em relação ao sexo, quando compararam os preços recebidos, os autores verificaram que as mulheres recebem preços mais altos pela commodity por reterem o produto consigo por um período maior à procura de melhores preços.

Glaser e Weber (2007) encontraram que investidores que acreditam ser mais habilidosos que a média, em termos de aplicações em investimentos, tendem a realizar mais negócios que os demais. Umarov e Sherrick (2005) identificaram sinais de excesso de confiança entre produtores e afirmaram que isso pode explicar a decisão de adquirir ou não seguros de produção de milho e soja. Eales et al. (1990) também acharam sinais de excesso de confiança entre produtores de soja quando analisaram suas distribuições subjetivas de probabilidade para preços futuros e de opções.

Apesar de um crescente número de estudos analisarem seus resultados como excesso de confiança, de acordo com Glaser e Weber (2007), ainda não há uma definição precisa para esta expressão na literatura. Biais et al. (2005) definiram excesso de confiança como a tendência permanente dos indivíduos em superestimar suas habilidades, a probabilidade de resultados positivos, ou até mesmo a precisão de seu conhecimento.

Graham, Harvey e Huang (2005) distinguiram duas definições para o termo: uma utilizada em Finanças e a outra em Psicologia. Por um lado, na literatura de Finanças, o excesso de confiança geralmente ocorre quando há superestimação da precisão da informação relativa a um valor. Por outro, na literatura da Psicologia, o excesso de confiança pode significar tanto que o indivíduo acredita que sua distribuição subjetiva é mais compacta do que ela realmente é, ou ainda, que acredita que suas habilidades são maiores do que realmente são (GRAHAM, HARVEY e HUANG, 2005).

Mais precisamente, o excesso de confiança pode se manifestar em qualquer uma das seguintes formas: descalibragem, efeito melhor que a média, e ilusão de controle ${ }^{7}$.

7 Outras formas de excesso de confiança também podem ser listadas, tal como a estimação de volatilidades (GLASER e WEBER, 2007). Entretanto, algumas destas outras formas podem ser consideradas como definições alternativas ou parte das formas que são aqui apresentadas. 


\subsection{Descalibragem}

Eales et al. (1990) discutem sucintamente algumas razões pelas quais as expectativas individuais podem não coincidir com preços futuros e de opções. Uma das explicações dada por estes autores é que o cálculo das probabilidades individuais pode ser descalibrado com os resultados efetivos. Isto é, a expectativa do produtor pode não ser muito precisa para determinar a distribuição efetiva dos resultados. Por essa razão, é esperado que indivíduos com excesso de confiança tenham distribuições de probabilidades subjetivas mais compactas, ou seja, com menor variância.

Umarov e Sherrick (2005) descreveram duas maneiras diferentes para se determinar a presença de descalibragem entre indivíduos. A primeira é conhecida como o método do intervalo de confiança. Este método consiste em uma lista de questões, nas quais o entrevistado responde e aponta o quanto está confiante que sua resposta está correta. O nível de confiança do entrevistado é, então, comparado à probabilidade de se responder a resposta corretamente. Por exemplo, Biais et al. (2005) usaram este método para medir o grau de excesso de confiança de 245 participantes de um jogo de negócios. Foi pedido aos respondentes que apontassem valores para algumas questões gerais, tais como a idade de Martin Luther King Jr. quando faleceu, a distância aérea entre Londres e Tóquio etc.

A segunda maneira de se medir a descalibragem é pedir ao entrevistado que forneça sua distribuição de probabilidades subjetiva para uma quantidade/ produtividade/preço desconhecido. Esta distribuição subjetiva é então comparada à distribuição verdadeira ou histórica (distribuição objetiva). Se os intervalos indicados forem muito compactos, a conclusão é que a volatilidade histórica é subestimada e o investidor tem excesso de confiança.

Pease (1992), por exemplo, usou esse método para extrair as respostas sobre as probabilidades subjetivas das produtividades de 90 produtores de milho e soja no estado do Kentucky, Estados Unidos. Em um trabalho anterior, Eales et al. (1990) utilizaram o mesmo método para obter estimativas de expectativas de preços para diferentes grupos de fazendeiros e elevators no estado de Illinois, também nos Estados Unidos. O número de respondentes dentro de cada grupo variou entre 14 e 59.

De Bondt (1998) pediu a cada um dos 45 investidores que apontassem um único valor para fechamento do índice Dow Jones e para as principais ações que compõem o índice. Eles também foram solicitados a apresentarem intervalos estimados de preços para os quais eles acreditavam ter apenas uma chance em dez que o valor da ação pudesse subir e cair. $\mathrm{O}$ autor encontrou que os intervalos de confiança apontados eram muito compactos em relação à variabilidade atual de preços.

Smith e Mandac (1995) estimaram e compararam as probabilidades subjetivas e objetivas dos rendimentos de arroz de produtores nas Filipinas. As entrevistas 
foram aplicadas a 21 fazendeiros de duas diferentes regiões produtoras no país. O objetivo da pesquisa foi analisar se as distribuições objetivas poderiam ser consideradas aproximações razoáveis das percepções dos produtores. As entrevistas foram realizadas antes que os produtores tivessem qualquer previsão das condições do tempo para a safra daquele mesmo ano. Primeiramente, os autores pediram aos produtores que especificassem suas previsões para o menor e pior rendimento na próxima safra. Esta amplitude foi então dividida em cinco intervalos iguais. Vinte e cinco moedas (o que era equivalente à quantia paga por um dia de trabalho) foram dadas aos produtores e foi solicitado a eles que as distribuíssem entre os intervalos. Os produtores foram informados que, ao final da safra, os rendimentos dos talhões de arroz seriam medidos, e eles receberiam a quantidade de moedas alocadas no intervalo no qual o rendimento realizado estivesse contido. Eles encontraram que, enquanto as médias foram similares, as variâncias subjetivas foram substancialmente inferiores às variâncias objetivas.

\subsection{Efeito melhor que a média}

Alguns trabalhos sugerem que investidores podem ter excesso de confiança se acreditarem que eles se encontram acima da média, isto é, se eles se julgam pelo menos tão bons quanto outros em relação a suas habilidades ou atributos pessoais. (GLASER e WEBER, 2007).

Benoît e Dubra (2008) mencionaram alguns trabalhos nos quais o efeito melhor que a média é descrito, mostrando que muitas pessoas consideram-se mais inteligentes que a média, muitos gerentes financeiros consideram seus desempenhos melhores que os de seus colegas, ou que muitos estudantes de ensino médio consideram-se mais originais do que os demais estudantes.

Tanto Glaser e Weber (2007) quanto Benoît e Dubra (2008) apontaram o trabalho desenvolvido por Svenson (1981) com um dos clássicos exemplos dados ao efeito melhor que a média. No trabalho de Svenson (1981), os entrevistados foram perguntados a respeito de suas competências como motoristas em relação a um determinado grupo. Ele encontrou que $77 \%$ dos entrevistados suecos declararam-se motoristas mais seguros que a média, e $69 \%$ consideraram-se mais habilidosos. Ele ainda encontrou que, entre um grupo de estudantes, $82 \%$ acreditavam estar ranqueados entre os 30\% motoristas mais seguros.

Utilizando informações de um questionário aplicado a 258 produtores de milho no estado de Illinois, Egelkraut et al. (2006) investigaram a relação entre os rendimentos subjetivos e objetivos e a relação destes com o uso de seguro de produtividade de milho. Entre outras motivações para o trabalho, foi incluída a observação de que, mesmo que os prêmios dos seguros fossem justos, existiria pouca motivação para que os fazendeiros utilizassem o seguro caso a distribuição subjetiva das produtividades fosse mais concentrada em valores altos. Baseados nas respostas aos questionários em relação à produtividade do 
milho e à percepção dos produtores de seus resultados relativos comparados a outros produtores, os autores encontraram que os produtores veem si mesmos como melhores que a média em relação à produtividade e à variabilidade da produção.

O trabalho desenvolvido por Hyytinen e Pajariten (2005) é outro exemplo destacado do efeito melhor que a média em finanças. Os autores realizaram a pesquisa com uma amostra de 393 firmas iniciantes no mercado, com até quatro meses de idade, na Finlândia. Uma das questões incluídas no questionário pedia aos empresários que estimassem a probabilidade de que o negócio pudesse falhar, de modo que eles não estariam mais em atividade nos próximos três anos, a partir daquela data. De acordo com os resultados, $87 \%$ dos empresários esperavam que seus novos negócios permanecessem pelo menos três anos em atividade. No entanto, as estatísticas apontavam, naquela época, que a taxa de sobrevivência era ao redor de $60 \%$.

\subsection{Ilusão de controle}

De acordo com Graham, Harvey e Huang (2005), o efeito de ilusão de controle pode ser definido como a tendência de alguém acreditar que sua estimativa da probabilidade de sucesso é maior que a probabilidade objetiva pode garantir.

Kee, Foo e Lim (2002) definiram o efeito de ilusão de controle como o viés no qual o indivíduo superestima a extensão na qual suas habilidades podem ser aumentadas em situações nas quais a sorte também pode determinar os resultados, e a habilidade não é o fator decisivo. Estes mesmos autores mencionaram que empresários geralmente mostram uma preferência pouco comum em exercer controle sobre seus resultados porque acreditam também exercer controle sobre pessoas e eventos. Consequentemente, indivíduos que exibem essa forma de excesso de confiança são mais propensos a subestimarem o risco porque acreditam que suas habilidades podem precaver o acontecimento de eventos negativos.

Após discutir algumas formas de excesso de confiança, Umarov e Sherrick (2005) fizeram a seguinte pergunta: é razoável esperar que exista excesso de confiança entre produtores? Eles responderam que, embora algumas formas de excesso de confiança (tais como o efeito melhor que a média e o otimismo) possam ser encontradas em várias ocupações, a descalibragem é um caso diferente porque varia entre profissões. De acordo com Gervais, Heaton e Odean (2002), na presença de alta previsibilidade, repetição de tarefas simples e quando o retorno é claro e rápido, os indivíduos são bem calibrados. Diante disto, podem ser classificados como bem calibrados os jogadores de baralho e jóqueis, por exemplo.

Quando analisadas as três condições listadas por Gervais, Heaton e Odean (2002), Umarov e Sherrick (2005) concluíram que existe evidência empírica que 
sugere a presença de excesso de confiança entre produtores agropecuários. Concluíram isso pois a previsibilidade dos resultados da colheita é baixa; os preços e as produtividades são variáveis aleatórias; o retorno ao investimento inicial é distante no tempo e pouco preciso. Além disso, as decisões dos produtores são, de certa forma, complicadas e de aprendizagem complexa, podendo levar ao viés de excesso de confiança nos seus julgamentos. De acordo com Umarov e Sherrick (2005), a profissão de produtor poderia estar enquadrada em algum ponto entre a previsão do tempo e a análise de investimentos de capital.

\section{Metodologia}

\subsection{Modelo teórico}

Apesar de poder se manifestar de todas as maneiras descritas na seção anterior, Glaser e Weber (2007) afirmam que o excesso de confiança pode ser teoricamente modelado como uma falha na precisão da estimação, utilizando-se informações privadas.

Para entender melhor este modelo, considera-se um produtor que tenta prever o preço de venda, $\tilde{p}$, para seu produto, na próxima safra. Assume-se que o preço seja normalmente distribuído $\tilde{p} \sim N\left(\mu_{p}, \sigma_{p}^{2}\right)$. De acordo com Glaser e Weber (2007), o produtor recebe sinais privados $\tilde{s}$ a respeito do comportamento de $\tilde{p}$, descritos como $\tilde{s}=\tilde{p}+c \cdot \tilde{e}$, sendo que $\tilde{e}$ é uma variável aleatória normalmente distribuída $\left(\tilde{e} \sim N\left(0, \sigma_{e}^{2}\right)\right)$. As informações contidas em $\tilde{s}$ podem ser entendidas como informações históricas de preços, de produtividade de condições climática etc. Assume-se, ainda, que $\tilde{p}$ e $\tilde{e}$ são independentes de tal forma que $\tilde{s} \sim N\left(0, \sigma_{p}^{2}+c^{2} \cdot \sigma_{e}^{2}\right)$. Se $c=1$, o investidor é racional, pois a variável aleatória $\tilde{e}$ não faz parte do sinal que o mesmo recebe e, portanto, não é incorporada nas suas previsões de preço. Se $0 \leq c<1$, então o produtor possui excesso de confiança. Este excesso de confiança ocorre pelo fato de o produtor subestimar a variância condicional de $\tilde{p}$, dada a realização $s$, já ocorrida e, portanto, conhecida. A variância condicional de $\tilde{p}$ é representada da seguinte forma:

$$
\operatorname{Var}[\tilde{p} \mid \tilde{s}=s]=\operatorname{Var}(\tilde{p})-\frac{(\operatorname{Cov}[\tilde{p}, \tilde{s}])^{2}}{\operatorname{Var}[\tilde{s}]}=\sigma_{p}^{2}-\frac{\sigma_{p}^{4}}{\sigma_{p}^{2}+c^{2} \sigma_{e}^{2}}
$$

Outros autores, tais como Umarov e Sherrick (2005), também utilizaram este mesmo modelo para descrever e melhor compreender o processo pelo qual produtores subestimam a previsão da variância subjetiva quando são descalibrados. 


\subsection{Análise estatística dos questionários}

No correr de outubro e novembro de 2008, 90 produtores de milho no Sul e Centro-Oeste do Brasil foram entrevistados. Os respondentes do Sul do Brasil consistiram em novos e antigos clientes de uma empresa de consultoria (Noble Group), enquanto os respondentes do Centro-Oeste eram associados de um instituto socioeconômico (Instituto Mato-Grossense de Economia Agropecuária) ou produtores com alguma relação com a Universidade Estadual de Goiás.

A expectativa subjetiva de preços dos produtores foi obtida por meio de dois formatos diferentes. No primeiro, o formato direto, utilizando-se uma pergunta direta, as expectativas de preço físico e futuro foram obtidas simplesmente perguntando-se aos produtores qual o preço médio mais provável pelo qual esperavam vender a saca de $60 \mathrm{~kg}$ de milho, em março de 2009, no caso do mercado fisco; e qual seria a cotação mais provável da saca de milho de $60 \mathrm{~kg}$ no mercado futuro da BM\&FBovespa, nos vencimentos março e julho de 2009.

No segundo, o formato indireto, foi solicitado aos produtores que determinassem os valores das probabilidades que eles acreditavam que o preço do milho pudesse estar dentro dos intervalos de preços listados, em março e julho de 2009, dependendo da região onde o mesmo possui propriedade. Março e julho foram escolhidos primeiramente por serem os meses mais próximos da colheita no Sul e Centro-Oeste ${ }^{8}$ do Brasil, respectivamente. Em segundo lugar, representam os vencimentos com maior liquidez no mercado futuro de milho da BMF\&Bovespa.

A participação na pesquisa foi voluntária e sem assistência, e nenhum incentivo financeiro foi dado aos respondentes.

A mesma metodologia de pesquisa foi utilizada por Clop-Gallart e JuárezRubio (2007), Egelkraut et al. (2006) e Eales et al. (1990), na obtenção das probabilidades estimadas.

\subsection{A distribuição subjetiva de probabilidades e o método de comparação das volatilidades subjetivas de preços spot e futuro}

A probabilidade subjetiva pode ser interpretada como a expectativa do tomador de decisão a respeito do resultado de um evento não repetitivo e incerto (GRISLEY e KELLOG, 1983). Pode ser ainda compreendida como a tradução das expectativas de uma pessoa, após a mesma ter processado todas as informações disponíveis, utilizado suas habilidades. Além disso, o verdadeiro valor das probabilidades deve estar entre 0 e 1 . Considerando-se esta última afirmação, no momento de responder os questionários, os entrevistados foram solicitados que colocassem valores entre 0 e $100 \%$ para refletir suas percepções

8 Somente para o estado de Mato Grosso. Em Goiás, a primeira safra é a mais importante, assim como na região Sul. 
de probabilidade em cada um dos intervalos listados. Pease (1992) definiu uma condição de "coerência de probabilidade" como a exigência de que a soma das probabilidades listadas nos intervalos seja igual a $100 \%$.

Como o objetivo deste artigo é testar o excesso de confiança baseado no efeito de descalibragem, verificou-se, primeiramente, se os respondentes foram bem calibrados nas suas respostas referentes à estimação direta e indireta das expectativas de preços. Para realizar esta comparação, a literatura propõe a utilização do teste bicaudal t-Student, expresso da seguinte forma:

$$
t_{\alpha / 2}=\frac{P^{I}-P^{D}}{S_{P I}}
$$

Em que $P^{D}$ é o preço apontado diretamente, $P^{I}$ e $S_{P^{l}}$ são respectivamente o preço apontado indiretamente e o desvio padrão, calculado a partir da distribuição subjetiva extraída no questionário, e $\alpha$ é o nível de significância escolhido para aplicação do teste. A hipótese nula do teste é de que não existem diferenças entre os dois preços. Enquanto isso, na hipótese alternativa, afirma-se que os respondentes podem ser descalibrados na estimação direta dos preços por projetarem um preço maior ou menor quando comparado à média subjetiva da distribuição subjetiva de preços.

Buzby et al. (1994), por exemplo, também analisaram discrepâncias entre as distribuições históricas e subjetivas de produtividade para produtores no estado do Kentucky, nos Estados Unidos. De acordo com os resultados, existe uma correspondência entre as médias das duas distribuições, mas os produtores superestimam suas produtividades e subestimam os riscos.

Segundo, o estudo procurou identificar se os indivíduos são descalibrados quando sub(super)estimam as variâncias dos preços spot quando comparadas às variâncias históricas dos preços futuros e spot. Se a variância subjetiva é menor que a empírica (isto é, se a distribuição subjetiva dos preços spot é mais compacta que a distribuição histórica dos preços futuros e spot), pode-se concluir que os produtores não esperam que os preços variem tanto quanto variaram, em média, no passado.

Com a hipótese de que o logaritmo natural dos preços $\left(P_{t}\right)$ é normalmente distribuído com média $\mu$ e variância $\sigma^{2}$, esses parâmetros foram calculados da seguinte forma (LIMPERT, STAHEL e ABBT, 2001; EALES et al., 1990,):

$$
\begin{aligned}
& \mu=e^{\left(\mu+\sigma^{2} / 2\right)} \\
& \operatorname{var}\left(P_{t}\right)=e^{2 \mu+\sigma^{2}}\left(e^{\sigma^{2}}-1\right)
\end{aligned}
$$

As variâncias obtidas através dos questionários foram calculadas a partir das expectativas mensais dos preços. Assim, de modo a realizar a comparação proposta, médias e variâncias mensais foram também calculadas a partir dos dados históricos dos preços futuros e spot para cada estado analisado. 
De acordo com Berenson e Levine (1999), uma vez que se assume que uma variável é normalmente distribuída, o teste qui-quadrado, $\chi^{2}$, pode ser utilizado para representar a distribuição da variabilidade da amostra de dados e para testar se a variância calculada (ou o desvio padrão) é igual a um valor específico. O teste estatístico pode ser calculado da seguinte forma:

$$
\chi^{2}=\frac{(n-1) S^{2}}{\sigma^{2}}
$$

Para uma dada amostra de tamanho $n$ (número de intervalos preenchidos com valores de probabilidades subjetivas) o teste estatístico $\chi^{2}$ segue distribuição qui-quadrado com (n-1) graus de liberdade. $S^{2}$ e $\sigma^{2}$ são as variâncias calculadas a partir da amostra (variância subjetiva) e a variância populacional hipotética (variância histórica), respectivamente.

O teste $\chi^{2}$ para igualdade de variâncias pode ser tanto bicaudal como unicaudal, dependendo se a hipótese alternativa é não direcional ou direcional. Uma vez que os indivíduos podem possuir excesso de confiança ou baixa confiança, Eales et al. (1990) propuseram que um teste $\chi^{2}$ bicaudal fosse aplicado para verificar diferenças entre as variâncias de mercado e as variâncias subjetivas para preços de soja e milho.

As hipóteses nula e alternativa podem ser construídas da seguinte forma:

$$
\begin{aligned}
& H_{0}: S_{\text {subjetiva }}^{2}=\sigma_{\text {historica }}^{2} \\
& H_{A}: S_{\text {subjetiva }}^{2} \neq \sigma_{\text {historica }}^{2}
\end{aligned}
$$

A regra de decisão é a seguinte:

Rejeita-se $H 0\left\{\begin{array}{c}\text { se } \chi^{2}>\chi_{U}^{2} \\ \text { ou se } \chi^{2}<\chi_{L}^{2}\end{array}\right.$, caso contrário, não se rejeita $H_{0}$.

A primeira condição implica que a variância individual é maior que a variância de mercado, levando-se à conclusão de que o indivíduo possui baixa confiança. A segunda condição implica que a variância subjetiva obtida no questionário é significativamente inferior à variância do mercado, o que leva à conclusão de que o indivíduo possui excesso de confiança.

\subsection{Dados}

Além das informações obtidas a partir dos 90 questionários aplicados, preços futuros e spot de milho foram coletados na BM\&FBovespa e no Cepea (Centro de Estudos Avançados em Economia Aplicada) ${ }^{9}$, respectivamente, de janeiro

\footnotetext{
9 Os preços obtidos junto ao Cepea foram utilizados como sendo a média estadual para
} cada um dos cinco estados nos quais os questionários foram aplicados. 
de 2004 a outubro de 2008. O contrato futuro com vencimento mais próximo foi utilizado na análise de preços do mercado futuro. O período utilizado foi escolhido devido à disposição de dados no mercado físico.

Em função dos custos de se realizar uma pesquisa de abrangência nacional com o enfoque aqui apresentado, foi aplicado apenas um número limitado de questionários, não se garantindo, assim, uma amostra estatisticamente representativa de população dos produtores de milho brasileiros. No entanto, espera-se que o conjunto de respostas obtido forneça uma leitura do comportamento de produtores em relação a preços ainda não realizada na literatura nacional.

\section{Resultados e Discussão}

Entre os 90 respondentes, 52 são localizados no Sul do Brasil (58\%), sendo a maior parte deles no estado do Rio Grande do Sul (34), seguido pelo Paraná (12) e Santa Catarina (7). Na região do Centro-Oeste do Brasil, a maioria dos respondentes foi de Mato Grosso (29), seguido pelo estado de Goiás (8). De acordo com a Conab - Companhia Nacional de Abastecimento (2008), esses estados encontram-se entre os seis maiores produtores de milho no País.

Os respondentes foram também solicitados a responderem questões adicionais relacionadas aos seus conhecimentos do mercado futuro e temas relacionados. Um total de dez indivíduos respondeu que tinha conhecimento sobre mercados futuros e negociava estes contratos na BM\&FBovespa. No entanto, a maioria (72 indivíduos) apontou que apenas tinha conhecimento, mas não negociava contratos futuros na bolsa. O restante, apenas oito respondentes, afirmou não ter conhecimento sobre o mercado futuro.

Aos produtores que indicaram conhecer, mas não negociam contratos futuros na BM\&FBovespa, foi perguntado qual(is) a(s) razão(ões) por não negociarem. $75,5 \%$ dos produtores responderam que não negociavam por não possuírem informações suficientes sobre a negociação nos mercados futuros. Nove produtores afirmaram que consideram o mercado futuro arriscado (12\%); quatro não negociam por ser muito caro $(5,5 \%)$, e somente $7 \%$ responderam abertamente que não negociam devido aos ajustes diários, porque não possuem assistência, ou porque existe muita burocracia envolvida na negociação.

Os entrevistados foram perguntados a respeito de outras formas de negociação do seu produto, de modo a haver alguma indicação da utilização do mercado a termo, como uma alternativa à negociação no mercado futuro. Somente oito produtores $(9 \%)$ disseram ter vendido parte da sua produção antecipadamente e nenhum deles, até aquele momento, havia vendido toda a produção.

Quando perguntados se já realizaram algum outro investimento a não ser aquele realizado na produção de milho, 12\% (11 respondentes) listaram 
investimentos alternativos. Entre eles, quatro (33,3\%) tinham investimentos em imóveis, enquanto os demais estavam envolvidos em atividades variadas.

Todos os produtores que indicaram não ter conhecimento sobre os mercados futuros tiveram também produção esperada inferior à média dos respondentes do mesmo estado. Por outro lado, todos aqueles que indicaram ter conhecimento e utilizam os mercados futuros como ferramenta de proteção de preços foram produtores cuja produção esperada mostrou-se superior à média dos respondentes do mesmo estado.

Apenas quatro produtores, todos eles do Rio Grande do Sul, responderam que produzem milho transgênico. Um deles afirmou não ter conhecimento dos mercados futuros, um conhece e negocia na bolsa, enquanto os outros dois conhecem, mas não negociam.

Para a realização da análise de excesso de confiança, somente aqueles respondentes que puderam ser considerados coerentes ${ }^{10}$ enquanto responderam a questão relativa à estimação das probabilidades subjetivas foram selecionados (86 respondentes). Três produtores que não responderam a essa questão também foram eliminados da análise, assim como outros dois que concentraram toda sua expectativa em somente um intervalo de preços. Desta forma, 81 respondentes foram selecionados para análise.

A Tabela 1, a seguir, apresenta informações históricas de preços para os estados analisados e também para os mercados futuros. As informações regionais contidas nesta tabela são referentes às médias mensais de preços obtidos junto ao Cepea, enquanto as informações relacionadas aos preços futuros foram obtidas a partir das cotações do primeiro vencimento em aberto dos contratos futuros de milho, na BM\&FBovespa.

Tabela 1. Resumo estatístico dos preços históricos estaduais e nos mercados futuros: jan/2004 a out/2008

\begin{tabular}{ccccccc}
\hline & PR & RS & SC & GO & MT & MF \\
\hline Média & 18,16 & 19,89 & 20,29 & 16,45 & 11,62 & 21,04 \\
Desvio & 3,82 & 3,71 & 3,85 & 3,82 & 3,27 & 4,07 \\
Máximo & 28,93 & 28,80 & 31,20 & 28,50 & 22,31 & 33,27 \\
Mínimo & 12,01 & 12,46 & 13,55 & 11,71 & 8,00 & 14,35 \\
\hline
\end{tabular}

Legenda: PR: Paraná; RS: Rio Grande do Sul; SC: Santa Catarina; GO: Goiás; MT: Mato Grosso; MF: Mercados Futuros da BM\&FBovespa.

Fonte: Cepea (2008) e BM\&FBovespa (2008).

10 Segundo conceito apontado por Pease (1992), um indivíduo é dito coerente se a soma de suas probabilidades subjetivas for igual a um $(100 \%)$. 
A Tabela 2, por sua vez, apresenta um resumo estatístico dos preços obtidos através dos questionários a plicados a produtores nos diversos estados analisados. Os valores de média e desvio padrão contidos nesta tabela referem-se às médias dos valores informados pelos produtores entrevistados nos respectivos estados.

Comparando-se as tabelas 1 e 2, pode-se verificar que as médias das expectativas de preços reveladas pelos produtores dos estados do Sul foram inferiores às médias mensais históricas calculadas, enquanto que, para os produtores dos estados do Centro-Oeste, o inverso aconteceu. Verifica-se ainda que, em todos os casos, os desvios padrão obtidos a partir dos questionários foram inferiores aos históricos. Estes resultados sugerem a presença de excesso de confiança entre os respondentes. No entanto, uma análise mais detalhada das distribuições de preços deve ser feita, seguindo o que foi sugerido na metodologia proposta.

Tabela 2. Resumo estatístico das distribuições de preços esperados obtidas através dos questionários: dados baseados nas médias estaduais

\begin{tabular}{cccccc}
\hline & PR & RS & SC & GO & MT \\
\hline Média & 17,48 & 18,57 & 17,81 & 23,64 & 13,18 \\
Desvio & 1,71 & 2,08 & 1,82 & 1,59 & 1,27 \\
Máximo & 18,90 & 19,80 & 18,00 & 27,00 & 15,60 \\
Mínimo & 15,20 & 17,40 & 17,40 & 18,10 & 11,26 \\
\hline
\end{tabular}

Legenda: PR: Paraná; RS: Rio Grande do Sul; SC: Santa Catarina; GO: Goiás; MT: Mato Grosso.

Fonte: Dados da pesquisa (2008).

Como foi assumido que os preços futuros e spot seguem distribuição lognormal, o teste Jarque-Bera (JB) foi aplicado para identificar se o logaritmo natural dos preços é normalmente distribuído. De acordo com a Tabela 3, a hipótese nula do teste não pode ser rejeitada para os valores calculados pela estatística JB, ao nível de significância de 5\%, para nenhuma das séries analisadas. Desta forma, pode-se concluir que a suposição anteriormente feita sobre distribuição lognormal de preços é aceitável.

Tabela 3. Teste Jarque-Bera para normalidade dos preços futuro e spot

\begin{tabular}{ccccccc}
\hline & Futures & $\begin{array}{c}\text { Rio Grande } \\
\text { do Sul }\end{array}$ & $\begin{array}{c}\text { Santa } \\
\text { Catarina }\end{array}$ & Paraná & Goiás & $\begin{array}{c}\text { Mato } \\
\text { Grosso }\end{array}$ \\
\hline JB & 1,362 & 1,368 & 1,345 & 1,739 & 3,044 & 1,362 \\
P-Valor & $(0,506)$ & $(0,504)$ & $(0,510)$ & $(0,419)$ & $(0,218)$ & $(0,506)$ \\
\hline
\end{tabular}

Fonte: Resultados da pesquisa. 
Para testar se os respondentes mostraram-se descalibrados quando apontaram as expectativas de preços nas formas direta e indireta, o teste t-Student foi utilizado ${ }^{11}$. Os preços na equação (2) foram calculados como as médias de preços de suas respectivas distribuições lognormais pela equação (3), assim como as variâncias na equação (4). De acordo com os resultados, ao nível de significância de 5\%, a hipótese nula de igualdade entre os preços apontados nas formas direta e indireta não pode ser rejeitada para apenas sete respondentes $(8,64 \%) .74(91,35 \%)$ produtores revelaram-se descalibrados quando calculados e comparados os preços e os valores esperados das distribuições de preços apontados pelos mesmos. Todos os indivíduos do Sul do Brasil revelaram-se descalibrados. Seguindo Eales et al.(1990), as variâncias individuais subjetivas foram comparadas com as variâncias históricas. Os valores calculados das estatísticas qui-quadrado foram então comparados com os respectivos valores das estatísticas de teste bicaudal (5\% de significância) para, desta forma, avaliar se os indivíduos revelaram-se com baixa confiança ou com excesso de confiança.

A variância subjetiva de cada produtor foi comparada à variância histórica de seu respectivo preço regional. $44,4 \%$ dos produtores mostraram-se descalibrados, de modo que suas variâncias subjetivas subestimaram as variâncias históricas obtidas a partir dos preços regionais. $28(44,12 \%)$ desses produtores são do Sul do Brasil e oito $(28,57 \%)$ deles do Centro-Oeste.

Quando as variâncias subjetivas dos produtores foram comparadas às variâncias históricas dos preços futuros, 62 (75,54\%) entrevistados foram considerados descalibrados. 71,69\% dos produtores do Centro-Oeste e 85,71\% dos produtores do Sul do País apresentaram variâncias subjetivas estatisticamente inferiores às variâncias dos preços futuros.

Com a exceção de um produtor, todos os demais que responderam que tinham conhecimento e negociavam contratos futuros na BM\&FBovespa, mostram-se com excesso de confiança em relação às variâncias dos preços futuros. A maior parte $(66 \%)$ daqueles produtores que responderam que tinham conhecimento, mas não negociavam contratos futuros na BM\&FBovespa porque não tinham informação suficiente, foi também identificada como descalibrada.

Além disso, dois entre os oito produtores $(25 \%)$ que já haviam negociado parte de sua safra mostraram-se descalibrados, assim como dois entre os onze $(18 \%)$ que afirmaram ter um investimento alternativo à atividade agrícola.

11 Uma vez que apenas $25 \%$ dos entrevistados responderam a questão relativa à indicação do preço futuro médio esperado na bolsa, optou-se por não incluir esta questão na análise. 


\section{Conclusões}

Como um primeiro trabalho deste tipo na literatura brasileira de derivativos agropecuários, essa pesquisa procurou apresentar, investigar e discutir razões pelas quais tão poucos produtores utilizam o mercado futuro agropecuário de milho para proteção de variações de preços. A explicação mais importante apresentada pelo presente trabalho consiste na percepção que os produtores possuem a respeito da variância dos preços spot e futuro.

O questionário aplicado no Sul e Centro-Oeste do Brasil mostrou que somente $10 \%$ dos respondentes negociam contratos futuros na Bolsa de Valores, Mercadorias \& Futuros. A maior parte dos produtores revelou que conhece, mas não faz negócios na bolsa. A razão mais importante apontada para não negociarem foi que os produtores não possuem informação suficiente para fazerem esse tipo de negócio.

Como apontado por muitos autores, um importante fator na determinação do tipo e extensão do hedge com opções, futuros e outras ferramentas de gerenciamento de risco pode ser a diferença nas variâncias subjetivas. Se os produtores percebem que as variâncias dos preços no mercado físico e nos mercados futuros são menores do que historicamente são, podem existir menos incentivos para os mesmos realizarem proteção de preços através dos mercados futuros.

Quando analisadas as diferenças entre as variâncias subjetivas e as variâncias históricas dos preços futuros e spot, foram encontradas evidências de falta de conformidade entre a percepção dos respondentes e o mercado. Foi encontrado que a maior parte dos respondentes espera que variâncias inferiores à do mercado ocorram, o que aponta para sinais de excesso de confiança entre os produtores de milho entrevistados. Também foram encontradas diferenças entre os preços spot apontados no formato direto e indireto, o que corrobora a hipótese de que os produtores são descalibrados na avaliação dos preços da commodity que produzem.

Enquanto esse trabalho pode ser considerado inovador na literatura de derivativos agropecuários brasileira, ainda é apenas um passo inicial rumo a novas investigações que ainda podem ser realizadas. Somente quando trabalhos futuros fizerem este mesmo tipo de levantamento ao longo do tempo e os questionários forem aplicados a um número maior de produtores, conclusões mais gerais poderão ser realizadas.

\section{Referências Bibliográficas}

AGUIAR, D. R. D Mercados Futuros e a Gestão do Risco nos Sistemas Agroindustriais Brasileiro. In: Workshop Brasileiro de Gestão de Sistemas Agroalimentares, 2., 1999. Anais... Ribeirão Preto: USP/Ribeirão Preto, 1999. p. 129-136. 
ALVES, J. S.; DUARTE S. G.; LIMA, R. C. testes da eficiência do mercado futuro do álcool anidro no Brasil: uma análise de co-integração. Revista Econômica do Nordeste, Fortaleza, CE, v. 39, n.1, p. 173-184, jan/mar. 2008.

ANDRADE, E. A. P. Mercados futuros: custos de transação associados à tributação, margem, ajustes e estrutura financeira. 2004. 115f. Dissertação (Mestrado em Economia Aplicada) - Escola Superior de Agricultura Luiz de Queiroz, Universidade de São Paulo. Piracicaba, SP, 2004.

ARBEX, M. A.; CARVALHO, V.D. Eficiência do Mercado Futuro de Café Brasileiro no período de 1992 a 1998. Revista de Economia e Sociologia Rural, Brasília, DF, v.37, n. 1, p. 97-113, jan/mar. 1999.

ASSOCIAÇÃO NACIONAL DOS CONFINADORES. Resultado do $1^{\circ}$ Censo de Confinadores de Goiás. Disponível em <http://www.assocon.com.br/not\%20 6\%202.htm >. Acesso em: 23 nov. 2008.

BENOÎT, J-P; DUBRA, J. Overconfidence? Munich: Munich, may 2008, 43 p. (Working Paper, 765).

BERENSON, M. L; LEVINE, D. M. Basic Business Statistics: Concepts and Applications, 7 ed. Upper Saddle River, NJ: Prentice-Hall, Inc. 1999. 1152 p.

BIAIS, B.; HILTON, D.; POUGET, S.; MAZURIER, K. Judgemental, overconfidence, self- monitoring and trading perfomance in an experimental financial market. The Review of Economic Studies. London, v. 72, p. 287-312, apr. 2005.

BODIE, Z.; KANE, A; MARCUS, A. J. Investiments. $5^{\text {th }}$ ed. New York: McGraw-Hill, 2001

BORELLA, J. B. O hedge utilizando contratos futuros como estratégia de gestão de risco de preço da soja: estudo de caso da cooperativa tritícola mista Alto Jacuí LTDA. 2004. 70f. Dissertação (Mestrado profissional em Economia). Universidade Federal do Rio Grande do Sul. Porto Alegre, RS, 2004.

BUZBY J.; KENKEL P.; SKEES J.; PEASE J.; BENSON F. A comparisson of subjective and historical yield distributions with implications for multiple period crop insurance. Agricultural Finance Review, Ithaca, NY, v. 54.p. 32-54, aug 1994.

CLOP-GALLART, M. M.; JUÁREZ-RUBIO, F. Elicitation of subjective crop yield PDF. Spanish Journal of Agricultural Research, Madrid, v.5, n. 1, p.16-24, 2007.

COMPANHIA NACIONAL DE ABASTECIMENTO. Brazilian Crop Assessment: Grains, 2008/2009. Disponível em: <www.conab.gov.br> . Acesso em: 23 nov. 2008.

CRUZ JUNIOR, J. C.; MARQUES, P. V.; MARTINES FILHO, J. G. Razão de hedge ótima de mínimo MPI (momento parcial inferior) no mercado futuro de boi 
gordo na BM\&F. In: CONGRESSO DA SOBER, 46., 2008, Rio Branco. Anais... Rio Branco: Sober. 2008. p. 1-18.

CUNNINGHAM III, L. T.; BRORSEN B. W.; ANDERSON, K. B.; TOSTÃO, E. 2008. Gender differences in marketing styles. Agricultural Economics, Chicago, v. 38: 1-7, oct. 2008.

DE BONDT, W. F. M. A portrait of individual investor. European Economic Review, London, v. 42, p. 831-844, may 1998.

EALES, J. S.; ENGEL, B. K.; HAUSER, R. J., THOMPSON, S. R. Grain price expectations of Illinois farmers and grain merchandisers. American Journal of Agricultural Economics, Chicago, v. 72, n. 1, p.701-708, mar. 1990.

EGELKRAUT, T.; B. SHERRICK, P. GARCIA; PENNINGS, J. 2006. Producers' yield and yield risk: perceptions vs. reality. Long Beach: American Agricultural Economics Association, 2006. Disponível em: < http://ageconsearch.umn.edu/ handle/21369>. Acesso em: 1 nov. 2006.

GERVAIS, S.; HEATON, J.; ODEAN, T. The Positive Role of Overconfidence and Optimism in Investment Policy, Philadelphia: Wharton Business School. feb. 2002, 49 p. (Working Paper 15-02).

GLASER, M.; WEBER, M. Overconfidence and trading volume. Geneva Risk Insurance Review. Geneva, v. 32, p. 1-36, july 2007.

GRAHAM, J. R.; HARVEY; C. R.; H.; HUANG, H. Investor Competence, Trading frequency, and home bias. Cambridge: National Bureau of Economic Research, june. 2005. (Working Paper, 114426).

GRISLEY, W.; KELLOG, E. D. Farmers' subjective probabilities in Northern Thailand: an elicitation analysis. American Journal of Agricultural Economics, Chicago, v. 65, n. 1, p. 74-82, feb. 1983.

HYYTINEN A.; PAJARITEN, M. Why are all new entrepreneurs better than average? Evidence from subjective failure rate expectations. Helsinki: The Research Institute of the Finnish Economy, june. 2005. 41p. (Discussion Paper, 987).

HULL, J. Fundamentos dos mercados futuros e de opções. Tradução de Marco Aurélio Teixeira. 4. ed. São Paulo: Bolsa de Mercadorias \& Futuros, 2005. 340 p.

INSTITUTO BRASILEIRO DE GEOGRAFIA E ESTATÍSTICA - IBGE. Banco de Dados Agregados - Sistema IBGE de Recuperação Automática - SIDRA. Disponível em: < http://www.ibge.gov.br>. Acesso em 23 nov. 2008.

KEH, H. T.; FOO, M. D.; LIM, B. C. Opportunity Evaluation under Risky Conditions: The Cognitive Processes of Entrepreneurs. Entrepreneurship Theory and Practice, Waco, TX, v. 27, n.2, p. 125-148, Winter 2002. 
LIMPERT E., STAHEL W; ABBT M. Log-normal Distributions across the Sciences: Keys and Clues. BioScience, Washington D. C., v. 51, n.5, p. 341-352. 2001.

MARQUES, P. V.; MELLO, P. C.; MARTINES FILHO, J. G. Mercados Futuros e de Opções Agropecuários - exemplos e aplicações para os mercados brasileiros. 2. ed. Rio de Janeiro: Elsevier, 2008. 223 p.

PEASE, J.W. A Comparison of Subjective and Historical Crop Yield Probability Distributions. Southern Journal of Agricultural Economics, Atlanta, v. 24, n. 2, p. 23-32, dec. 1992.

SMITH, J.; MANDAC, A. M. Subjective versus objective yield distributions as measures of production risk. American Journal of Agricultural Economics, Chicago, v. 77, n.1, p. 152-161. 1995.

SILVEIRA, R. L. F; FERREIRA FILHO, J. B. S. Análise das Operações de CrossHedge do Bezerro e do Hedge de Boi Gordo no Mercado Futuro da BM\&F. Revista de Economia e Sociologia Rural, Brasília, DF, v.41, n.4, p. 881-899, nov. 2003.

SVENSON, O. Are We All Less Risky and More Skillful Than Our Fellow Drivers? Acta Psychologica, New York, NJ, v. 47. n. 2, feb. 1981.

UMAROV, A.; B. J.SHERRICK, B. J. Farmers' subjective yield distributions:calibration and implications for crop insurance valuation, Providence: American Agricultural Economics Association. Disponível em: < http://ageconsearch.umn.edu/handle/19396>. Acesso em: 1 nov. 2005. 


\section{Exemplo de Questionário Aplicado na Regiâo Sul do Brasil}

Bom dia/Boa tarde, gostaria de pedir a gentileza de sua colaboração para a pesquisa da Esalq/USP e da Universidade de Illinois (EUA). A pesquisa é breve e consiste em apenas nove perguntas diretas e não deve durar mais de três minutos.

(1) Em qual localidade o sr.(a) possui propriedade? Que tipo de milho produz $(\mathrm{C}=$ convencional ou $\mathrm{T}=$ transgênico $)$ ?

Cidade:

Estado:

Tipo de milho:

(2) Você conhece ou usa a BM\&FBovespa?

( ) Não conheço

( ) Conheço e uso

( ) Conheço, mas não uso

(3) Se você conhece, mas não usa os mercados futuros, qual o motivo por não utilizar?

( ) É muito arriscado

( ) É muito caro

( ) Não tenho informação suficiente/não conheço bem

( ) Outro:

(4) Qual a sua produção esperada de milho para esta safra 2008-2009 (somente safra)?

$\mathrm{R}$ :

(5) Já realizou a venda antecipada (parcial ou integral) da sua safra?
( ) Não
( ) Parcial
( ) Integral

(6) Você possui outros investimentos fora do agronegócio? Em caso afirmativo, cite ao menos o principal.

( ) Não
( ) Sim 
(7) Qual o preço médio mais provável pelo qual você espera vender a saca de milho $(60 \mathrm{~kg})$, em março/2009, na sua região? $\mathrm{R}:$

(8) Qual o preço futuro médio esperado, na BM\&FBovespa, para a saca de milho de $60 \mathrm{~kg}$, em março de 2009 ?

R:

(9) De acordo com sua experiência e expectativas, preencha os espaços abaixo com valores da chance (em \%) do preço médio de venda da saca de milho $(60 \mathrm{~kg})$ estar em um destes intervalos, em março de 2009. Valores em $\mathbf{R} \$$ /sc $60 \mathrm{~kg}$ (NÃO É NECESSÁRIO PREENCHER TODOS OS ESPAÇOS).

\begin{tabular}{|l|l|l|l|l|l|l|l|l|l|}
\hline & & & & & & & & & \\
\hline $9-11$ & $11-13$ & $13-15$ & $15-17$ & $17-19$ & $19-21$ & $21-23$ & $23-25$ & $25-27$ & $27-30$ \\
\hline
\end{tabular}

